\title{
Experimentando a Multidisciplinaridade no Desenvolvimento de Apps
}

\author{
Daniela F. Brauner ${ }^{1}$, Raquel Janissek-Muniz ${ }^{1}$, Lisandro Zambenedetti Granville ${ }^{3,4}$, \\ Karina Moura ${ }^{1}$, Sandro Fetter ${ }^{2}$, Gabriel Nazar ${ }^{3,4}$ \\ ${ }^{1}$ Escola de Administração UFRGS - Departamento de Ciências Administrativas \\ ${ }^{2}$ Escola de Arquitetura UFRGS - Departamento de Design e Expressão Gráfica \\ ${ }^{3}$ Instituto de Informática UFRGS - Departamento de Informática Aplicada \\ ${ }^{4}$ Sociedade Brasileira de Computação (SBC) \\ Porto Alegre, RS - Brasil \\ \{daniela.brauner, rjmuniz, sandro.fetter, lisandro.granville\}@ufrgs.br
}

\begin{abstract}
This paper presents the App Challenge UFRGS 2016, a hackathon-style event, promoted to integrate students' expertise in Administration, Design and Computing. Combining students in multidisciplinary teams, the approach was to present a challenge to the development of mobile applications (Apps) over a weekend, including prototyping, business modeling and validation. The results demonstrate that multidisciplinary teams, focusing on viable products, can contribute in a complementary and collaborative way in the development of apps.
\end{abstract}

Resumo. Este artigo relata o App Challenge UFRGS 2016, um evento no estilo hackathon, promovido para integrar expertises de alunos de Administração, Design, e Computação. Combinando os estudantes em equipes multidisciplinares, a abordagem foi apresentar um desafio de desenvolvimento de aplicativos para dispositivos móveis (Apps) durante um final de semana, incluindo prototipagem, modelagem do negócio e validação. Os resultados demonstram que times multidisciplinares usando uma abordagem com foco em produtos viáveis podem contribuir de forma complementar e colaborativa no desenvolvimento de aplicativos.

\section{Introdução}

As modernas práticas de gestão vêm transformando as formas de trabalho no desenvolvimento de software. A adoção de métodos ágeis para gestão de projetos (FRISHBERG; LAMBDIN, 2015), as técnicas de modelagem de problemas via Design Thinking (BROWN, 2010) e seu uso para levantamento de requisitos (BERNAL, 2016) e a prototipagem rápida de mínimos produtos viáveis (CAROLI, 2015) exigem que os profissionais, em geral da computação, sejam flexíveis e multifuncionais. Num mesmo projeto, além de atender às demandas técnicas (de desenvolvimento, teste e operação de infraestrutura, atividades típicas de profissionais de TI), os membros dos times também 
devem realizar a elicitação de requisitos junto aos clientes, compreensão e modelagem do mercado e do negócio, bem como a validação do produto resultante. Tarefas estas que poderiam ser delegadas a pessoas com formações complementares.

Um hackathon é um evento de programação em Computação focado em problemas (TOPI e TUCKER, 2014) que encoraja a experimentação e a criatividade, aspectos essenciais à inovação (BRISCOE e MULLIGAN, 2014). Hackathons vêm sendo promovidos com bastante frequência em universidades, empresas ou como desafios de órgãos públicos, como forma de engajar times de desenvolvimento de forma intensa e desafiadora durante um curto período de tempo. A abordagem de envolver múltiplas competências nos times dos hackathons é relativamente nova (BRAUNER et. al, 2016), visto que anteriormente apenas profissionais da Computação eram envolvidos na maioria dos eventos do tipo.

Com base nas experiências anteriores e no intuito de testar e evoluir a aplicação de técnicas de desenvolvimento de software com times multidisciplinares, este artigo descreve a experiência do App Challenge UFRGS 2016. Na seção 2, é apresentado o modelo do evento, etapas e critérios utilizados. A seção 3 relata o processo base de desenvolvimento apresentado como sugestão aos times de desenvolvimento. Os resultados são apresentados na seção 4. Para finalizar, a seção 5 apresenta as conclusões e considerações futuras.

\section{O Modelo do Evento}

O App Challenge UFRGS 2016 ofereceu aos alunos um desafio durante um final de semana, incluindo prototipagem, modelagem do negócio e validação. A abordagem foi a de agrupar alunos da UFRGS em equipes multidisciplinares para transformar ideias submetidas pela comunidade em soluções inovadoras baseadas em Apps com planos de negócio. As equipes, acompanhadas por professores e mentores, exploraram a experimentação prática e multidisciplinar, o trabalho colaborativo e a contribuição social a partir em duas trilhas temáticas: segurança pública e impacto social. O evento ocorreu de 30 de setembro a 2 de outubro de 2016, e seguindo o modelo apresentado na Figura 1.

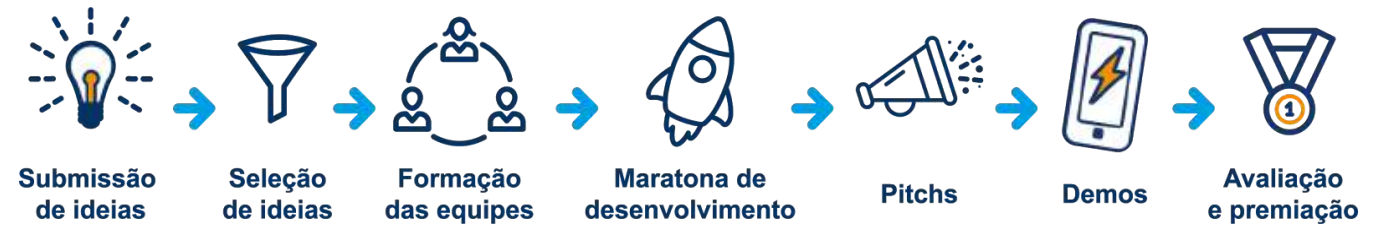

Figura 1: Modelo representando a dinâmica do evento. 
As etapas do modelo apresentado na Figura 1 são:

- Submissão de ideias - Chamada pública para submissão de ideias em duas trilhas temáticas: Segurança Pública e Impacto Social.

- Seleção de ideias - As ideias foram selecionadas por um comitê de professores, alunos e profissionais das áreas envolvidas no evento. A avaliação utilizou critérios como criatividade e inovação, viabilidade de implementação, viabilidade de negócio, impacto (social, ambiental ou econômico) e clareza.

- Inscrições dos participantes e formação das equipes - Alunos matriculados nos cursos de Administração, Design, Ciência e Engenharia da Computação (graduação e pós-graduação) se inscreveram no evento, informando, por ordem de preferência, as ideias que desejavam participar. As equipes foram organizadas previamente, incluindo alunos de todas as áreas, priorizando as inscrições por ordem de chegada e preferências de ideias indicadas pelos alunos.

- Maratona de desenvolvimento - A Maratona ocorreu no Nós Coworking que forneceu a estrutura necessária, atuando como co-realizador do evento. O primeiro dia iniciou com palestras e foi seguido pela formação dos times, iniciando os elementos estruturadores para ideação das suas soluções. A partir do $2^{\circ}$ dia (sábado), as equipes retornaram ao local para desenvolvimento das ideias. Os alunos puderam permanecer no local durante a madrugada, se assim desejassem, até o dia seguinte ( $3^{\circ}$ e último dia do evento). Professores também permaneceram no local.

- Apresentação dos Pitchs - Pitchs de 5 minutos foram apresentados pelos times e avaliados por um comitê formado por professores e profissionais das áreas, além de representantes das entidades envolvidas na realização e empresas patrocinadoras.

- Demonstrações - O comitê de avaliação visitou os espaços de trabalho de cada equipe, podendo questionar as equipes e utilizar os protótipos desenvolvidos.

- Avaliação e premiação - O comitê se reuniu e elegeu uma equipe vencedora para cada trilha, baseando-se nos pitchs e na avaliação dos protótipos. O comitê discutiu os resultados subjetivamente com base nos seguintes critérios: (i) Inovação: quanto às soluções propostas (negócio, design e tecnológicas); (ii) Organização: quanto à gestão interna do time durante o desafio relacionado à gerenciamento das atividades, 
processo de desenvolvimento e escopo atingido; (iii) Qualidade: quanto ao protótipo desenvolvido, identidade visual e modelagem do negócio; (iv) Apresentação; e (v) Viabilidade de tornar-se um produto.

\section{Processo de Desenvolvimento Sugerido}

Com o passar dos anos, as técnicas de desenvolvimento de software tem evoluído. Desde o modelo em cascata, onde o software era construído de forma sequencial, até as atuais metodologias ágeis, onde o software é incrementalmente evoluído (SOMMERVILLE, 2011). As modernas técnicas de modelagem de problemas via Design Thinking (BROWN, 2010) vêm sendo aplicadas para levantamento de requisitos (BERNAL, 2016), trazendo contribuições importantes para os times de desenvolvimento. A prototipagem rápida de mínimos produtos viáveis (CAROLI, 2015), para validação dos protótipos intermediários em conjunto com metodologias ágeis de desenvolvimento de software (SCHWABER e BEEDLE, 2002), exige que os profissionais, em geral da Computação, sejam flexíveis e multifuncionais.

Neste contexto, o primeiro dia do evento iniciou com palestras introdutórias sobre os principais conceitos que englobam o processo de desenvolvimento sugerido. Entre eles: UX (User Experience), mostrando a importância de modelar o comportamento dos usuários para mapear suas necessidades reais; Cultura de desenvolvimento ágil, mostrando a importância do foco na entrega em ciclos curtos de desenvolvimento; e, MVP (Minimum Viable Product) e suas funcionalidades, mostrando a importância de planejar entregas pequenas e viáveis, pensando sempre na relevância do entregável sob a perspectiva do usuário, qualidade e viabilidade. Não foi feita uma palestra sobre Business Model Canvas ou BM Canvas (OSTERWALDER e PIGNEUR, 2009), embora ele seja parte do processo proposto, devido a disponibilidade de mentores no tema ao longo de todo evento.

Para consolidar o modelo proposto, foram apresentados aos times de desenvolvimento um conjunto de materiais e disponibilizados mentores especializados nos temas, para que os times pudessem adotar uma metodologia de desenvolvimento ágil baseada nos componentes propostos. O modelo é brevemente apresentados na Figura 2. 


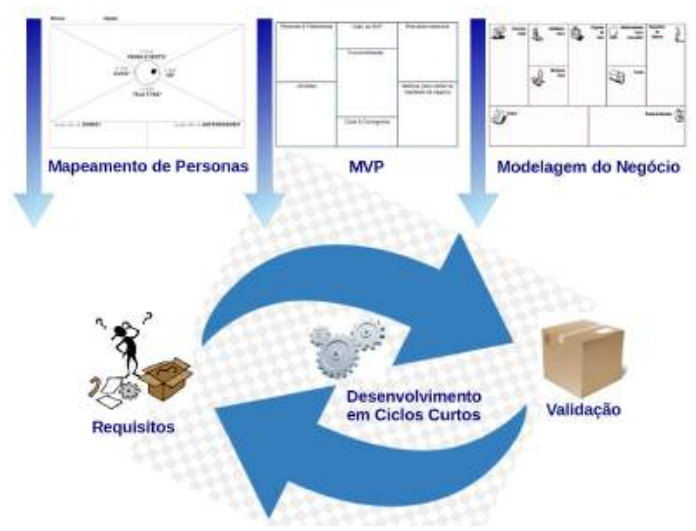

Figura 2: Processo de desenvolvimento sugerido.

A metodologia sugerida inicia pelo mapeamento personas relacionadas à ideia. Todos os componentes dos grupos trabalharam no mapa de empatia (Gray et al., 2010). A partir daí os times foram orientados a preencher uma versão inicial do Canvas MVP (CAROLI, 2015), para que planejassem um mínimo produto viável. Nessa etapa, é essencial a presença de pelo menos um dos programadores e um dos designers, para que as funcionalidades mínimas sejam validadas tecnicamente dentro do prazo de desenvolvimento, mas a presença dos administradores é essencial para garantir que tais funcionalidades atendam à validação do negócio. Os administradores também foram essenciais para criação das métricas de validação, custos e cronograma. Os designers contribuíram sobremaneira na definição de jornadas de usuário, visto seus conhecimentos em User Experience. De posse dos requisitos, os designers e programadores partiram para o desenvolvimento do primeira versão do protótipo que atendesse ao MVP planejado. Enquanto isso, os administradores partiram para a modelagem do negócio, usando o modelo do Business Model Canvas (BM Canvas), buscando intervenções dos demais integrantes quando necessário. Ao finalizar o BM Canvas, os resultados foram compartilhados e validados entre todos os integrantes, de forma a realimentar os requisitos e auxiliar na validação do MVP.

Para validação, cada time determinou a sua, de forma mais pertinente ao foco de seus aplicativos. Muitos escolheram validar a proposta de negócio com foco nas funcionalidades do MVP, mesmo sem utilizar o protótipo criado, enquanto outros optaram por apresentar o protótipo e validar com base no uso e nas jornadas dos usuários já implementadas.

\section{Resultados}




\subsection{Execução}

Ao todo, foram submetidas 28 ideias, sendo $74,1 \%$ para a trilha de impacto social e 25,9\% para a trilha segurança pública. Como a submissão de ideias era pública, das ideias submetidas por alunos da Universidade, 33,3\% foram oriundas de estudantes do curso de Administração, 11,1\% de alunos da Computação e 7,4\% de Design. A seleção das ideias foi realizada elencando-se as 5 propostas mais bem avaliadas de cada uma das trilhas.

Durante o período de inscrições, obteve-se 75 inscrições de alunos da Universidade. As inscrições foram bem distribuídas quanto às áreas de atuação (nas 3 disciplinas envolvidas no evento) e quanto ao gênero dos inscritos apresentando uma pequena prevalência com relação aos inscritos do sexo masculino em 54,7\%. Ao todo, 53 alunos compareceram ao evento formando os dez times conforme apresentado na Figura 3. Supõe-se que a alta taxa de evasão (i.e., 30\%) foi devido à coincidência de datas com as eleições municipais, à gratuidade das inscrições (que implica em um baixo comprometimento de comparecimento) e à falta de informação sobre a dinâmica do evento, explicitando a necessidade de aprimoramento na divulgação do mesmo.

Durante todo o evento, tivemos 99 pessoas presentes, entre participantes das equipes, convidados, mentores, organizadores, palestrantes e representantes dos patrocinadores. Destes, 24 atuaram como mentores. Nas mesas, foram disponibilizados os modelos impressos relacionados ao processo de desenvolvimento sugerido. Os estudantes levaram computadores e softwares necessários para os desenvolvimentos.

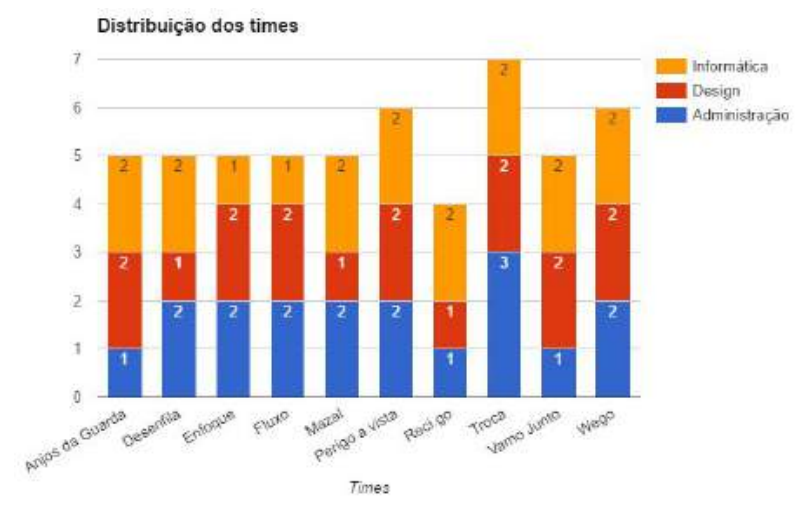

Figura 3: Distribuição dos participantes por time. 
Após os pitchs e visitações às demonstrações, a comissão de avaliação elencou um vencedor para cada trilha. Dentre os prêmios, um time recebeu vouchers para uso do espaço físico, com apoio para desenvolvimento do negócio oferecidos pelo coworking parceiro da realização do evento e o outro recebeu apoio especializado na organização do negócio e suporte para desenvolvimento da ideia junto à uma das incubadoras da Universidade, além de brindes e cursos oferecidos pelos patrocinadores e apoiadores. Todos os participantes das demais equipes receberam medalhas e outros prêmios relativos aos desafios promovidos ao longo do evento.

Com relação ao processo de desenvolvimento sugerido, $100 \%$ dos times adotaram em sua totalidade as ferramentas propostas e aplicaram o modelo conforme sugerido. Segundo relatos, em especial dos alunos da Computação, num mesmo projeto seguindo outra formação de time mais homogênea com colegas da Computação, eles precisariam atuar em todas as etapas, mesmo sem conhecimento. No processo proposto eles puderam focar em atender as demandas técnicas (de desenvolvimento, teste e operação de infraestrutura, atividades típicas de profissionais da área), e contribuíram na modelagem e validação dos resultados, aprendendo bastante com os colegas da administração e design. Segundo eles, dividir as tarefas de elicitação de requisitos, compreensão e modelagem do mercado e do negócio, bem como a validação do produto resultante, foi essencial para atingimento da meta: de obter um produto viável. Atividades de validação como a "pesquisa de campo" realizada por alguns grupos, foram essenciais para alteração de funcionalidades, melhorias nas jornadas de usuário e adequações do plano de negócios. Tarefas estas que foram delegadas a pessoas com formações complementares presentes nos times de desenvolvimento.

\subsection{Avaliação}

Ao final do evento, foi proposta uma atividade para promover o diálogo e a troca de experiências entre os participantes. Para isto, utilizou-se a técnica de fishbowl (MELO, 2013). A pergunta central foi "o que esse modelo de trabalho proporcionou para você e para sua profissão?”. Os depoimentos demonstraram que eventos deste tipo permitem aos estudantes uma maior compreensão do que é esperado deles no ambiente de trabalho, mostrando o quanto a universidade contribui para o conhecimento aplicado. Muitos depoimentos evidenciaram que a maioria dos estudantes ainda não tem certeza sobre seu desenvolvimento vocacional e área escolhida, mas a característica multidisciplinar das equipes viabilizou contribuições importantes, motivando os 
participantes com relação ao valor de seus conhecimentos e a valorização da sua profisssão. A possibilidade de compartilhar conhecimentos complementares nos times quebrou paradigmas entre as profissões e demonstrou a importância de se ter pessoas qualificadas em áreas diferentes para desenvolver um mesmo projeto.

Também foi criado um formulário Web que obteve 51 respostas. Na visão de $100 \%$ dos respondentes, o evento acrescentou conhecimentos relacionados à sua área de atuação. 58,6\% deles consideram que aprenderam muito sobre conceitos de outras áreas e $37,9 \%$ aprenderam algo. Em sua maioria, os participantes querem dar continuidade às ideias desenvolvidas no evento, onde apenas $7,1 \%$ não querem dar continuidade. Os demais elencaram como essenciais mais tempo, treinamento e mentoria.

A Figura 4 apresenta também a receptividade do modelo de formação dos times. Mais de 93\% dos respondentes gostaram de serem alocados em times onde não conheciam os outros integrantes. Em relação ao envolvimento das três áreas (Administração, Design e Informática), os respondentes foram unânimes na aceitação do modelo adotado. Além disso, entre os comentários, haviam sugestões de envolvimento de outras áreas em futuras edições do evento.

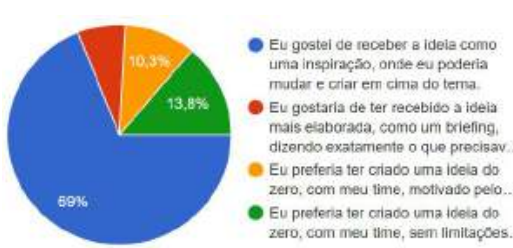

(a)

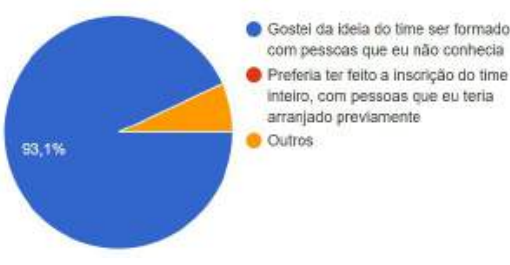

(b)

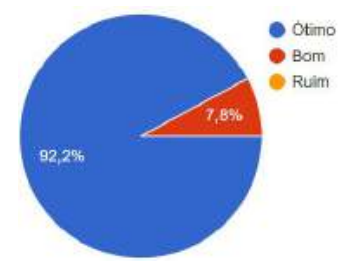

(c)

Figura 4: Receptividade do modelo do evento: (a) sobre as ideias, (b) sobre a formação dos times e (c) sobre o envolvimento das três áreas.

\section{Conclusões}

Este artigo apresentou o App Challenge UFRGS 2016, um evento realizado com o objetivo de promover uma experiência universitária multidisciplinar e aplicada. Além dos objetivos propostos pelo desafio de gerar aplicativos viáveis, o evento buscou apresentar aos participantes a importância e o impacto do trabalho multidisciplinar.

As equipes foram encorajadas a trabalhar compartilhando suas habilidades e conhecimentos específicos no desenvolvimento dos aplicativos. Ainda que muitos dos participantes não tivessem noções anteriores sobre desenvolvimento de software, a 
multidisciplinaridade entre os componentes provou a importância de se ter perfis complementares para o desenvolvimento e a validação de um produto mínimo viável.

Para os estudantes, passar por esta experiência de planejamento, execução e apresentação de um produto foi uma oportunidade tanto de validação da escolha profissional, quanto de perceber as capacidades e habilidades desenvolvidas durante o processo de aprendizagem em sala de aula. Além disso, o trabalho em equipe, o tempo limitado e os desafios propostos exigiram dos alunos flexibilidade, adaptação e comprometimento para que, em equipe, pudessem obter um resultado satisfatório ao final do evento.

A atuação dos perfis multidisciplinares no processo de criação do produto, tal como o envolvimento dos administradores para a validação da viabilidade e modelagem do negócio, os designers no desenvolvimento da experiência de usuário e os programadores com as informações sobre viabilidade e potenciais dificuldades técnicas, teve um impacto positivo: os estudantes puderam perceber o valor de suas habilidades. $\mathrm{Na}$ formação de equipes com 2 profissionais de cada área, os membros tomaram decisões mais objetivas e seguras quanto às atividades e desenvolvimento do projeto. Ainda assim, outras experiências são necessárias para confirmar, com base numa quantidade maior de dados, a formação ideal dos grupos com tal interdisciplinaridade.

Como resultado, o processo de desenvolvimento sugerido foi adotado em sua totalidade pelos participantes, evidenciando que habilidades distintas podem contribuir sobremaneira no desenvolvimento de aplicativos. Alunos da administração contribuíram em especial no levantamento de requisitos, modelagem das funcionalidades iniciais e avaliação do protótipo desenvolvido, através de técnicas de Design Thinking como mapeamento de personas, perfis de clientes, modelagem do MVP e validação do negócio através do Business Model Canvas, devolvendo feedbacks importantes para a implementação dos aplicativos e modelagem de interação de usuário. Os profissionais de design contribuíram para o mapeamento dos perfis de clientes, apoiando no processo de levantamento de requisitos e MVP, através da modelagem de jornadas de usuário empregando técnicas de User Experience, além de auxiliarem na validação e modelagem do negócio. Os alunos de computação, para implementação dos protótipos, 
tiveram que modelar e desenvolver um amplo entendimento sobre clientes, demandas e suas jornadas, contribuindo sobremaneira na definição das funcionalidades possíveis de implementação no prazo estipulado, que atendessem às demandas do negócio.

Para o futuro, pretende-se aprimorar o modelo e aplicá-lo em outras cidades, universidades ou até mesmo em novos eventos na mesma universidade. Dentre as melhorias potenciais são: a criação de métricas para aferição do impacto do evento e do modelo na comunidade, encaminhar os resultados gerados para potenciais investidores e atores do ecossistema de empreendedorismo, e incluir ou variar as áreas envolvidas, seja dando uma temática ao evento ou apenas buscando agregar outras expertises técnicas.

\section{Referências}

BERNAL, G. (2016) Especificação de requisitos de sistemas inteligentes assistidos: uma abordagem design thinking. Trabalho de Conclusão de Curso. Universidade Federal do Rio grande do Sul.

BRAUNER, D.F.; MARGREFF, P.; TAVARES, T.; da COSTA, V. K.; SILVA, A. (2016) Estímulo à prática multidisciplinar no ensino de Computação e Design através de um evento de programação focado em problemas. Proceedings of the WEI'2016 CSBC. Porto Alegre RS. Julho, 2016.

BRISCOE, G. and MULLIGAN, C. (2014) Digital innovation: The hackathon phenomenon. London: Creativeworks London Work Paper 6.

BROWN, T. (2010) Design Thinking - Uma metodologia poderosa para decretar o fim das velhas ideias. Rio de Janeiro: Elsevier, 2010.

BARDAGI, M. P.; LASSANCE, M. C. P.; PARADISO, A. C. (2003) Trajetória Acadêmica e Satisfação com a Escolha Profissional de Universitários em Meio de Curso. Revista Brasileira de Orientação Profissional, ISSN 1984-7270, SP, v.4, n.1-2, Dezembro, 2003.

CAROLI, P. (2015) Direto ao Ponto: Criando produtos de forma enxuta. São Paulo: Editora Casa do Código, 2015.

FRISHBERG, L.; LAMBDIN, C. (2015) Presumptive Design: Design Provocations for Innovation. New York: Elsevier Science \& Technology, 2015.

GONDIM (2002) Perfil profissional e mercado de trabalho: relação com a formação acadêmica pela perspectiva de estudantes universitários. Estudos de Psicologia 2002, 7(2), 299-309.

GRAY, D., BROWN, S. and MACANUFO, J. (2010). Gamestorming - A playbook for innovators, rulebreakers and changemakers. Sebastopol, CA: O’Reilly Media, Inc.

TOPI, H. and TUCKER, A. (2014) Computing Handbook. Third Edition: Information Systems and Information Technology. CRC Press, 2014.

IOWA UNIVERSITY (2015) SI Showcase: the basic collaborative learning techniques. Supplemental Instruction. Iowa State University. Iowa, USA, 2005.

KLEIN, J.T. (1990) Interdisciplinarity: History, theory, and practice. books.google.com

MELO, R.C. (2013) Collaborative Learning Topics for Teaching activities at Higher Technological Education in Tekhne e Logos, Botucatu, SP, v.4, n.2, Agosto, 2013.

OSTERWALDER, A. and PIGNEUR, Y., (2009) Business Model Generation, A Handbook for Visionaries, Game Changers, and Challengers. Amsterdam: OSF.

SCHWABER, K. and BEEDLE, M. (2002) Agile Software Development with Scrum. Upper Saddle River, NJ : Prentice-Hall. SOMMERVILLE, I. (2011) Engenharia de Software. $9^{\circ}$ edição. São Paulo, SP : Pearson Education do Brasil. 